\title{
On divisors of pseudovarieties generated by some classes of full transformation semigroups
}

\author{
Vítor H. Fernandes* \\ Departamento de Matemática \\ Faculdade de Ciências e Tecnologia \\ Universidade Nova de Lisboa \\ Monte da Caparica \\ 2829-516 Caparica \\ Portugal \\ E-mail: vhf@fct.unl.pt
}

\begin{abstract}
In this paper we present a division theorem for the pseudovariety of semigroups OD $[\mathrm{OR}]$ generated by all semigroups of order-preserving or order-reversing [orientationpreserving or orientation-reversing] full transformations on a finite chain.
\end{abstract}

2000 Mathematics Subject Classification: 20M20, $20 \mathrm{M} 07$.

Keywords: transformations, order-preserving, orientation-preserving, order-reversing, orientationreversing, pseudovarieties.

\section{Introduction and preliminaries}

In the 1987 "Szeged International Semigroup Colloquium" J.-E. Pin asked for an effective description of the pseudovariety (i.e. an algorithm to decide whether or not a finite semigroup belongs to the pseudovariety) of semigroups $O$ generated by all semigroups of order-preserving full transformations on a finite chain. This problem only had essential progresses after 1995. First, Higgins [23] proved that $\mathrm{O}$ is self-dual and does not contain all $\mathcal{R}$-trivial semigroups (and so $\mathrm{O}$ is properly contained in $\mathrm{A}$, the pseudovariety of all finite aperiodic semigroups), although every finite band belongs to O. Next, Vernitskii and Volkov [28] generalised Higgins's result by showing that every finite semigroup whose idempotents form an ideal is in $\mathrm{O}$ and in [11] the author proved that the pseudovariety of semigroups

* The author was (partially) supported by FCT and FEDER whithin the project POCTI-ISFL-1-143 of the Centro de Álgebra da Universidade de Lisboa. 
POI generated by all semigroups of injective order-preserving partial transformations on a finite chain is a (proper) subpseudovariety of $\mathrm{O}$. On the other hand, Almeida and Volkov [1] showed that the interval $[\mathrm{O}, \mathrm{A}]$ of the lattice of all pseudovarieties of semigroups has the cardinality of the continuum and Repnitskil and Volkov [27] proved that $\mathrm{O}$ is not finitely based. In fact, moreover, Repnitskiu and Volkov proved in [27] that any pseudovariety of semigroups $\mathrm{V}$ such that $\mathrm{POI} \subseteq \mathrm{V} \subseteq \mathrm{O} \vee \mathrm{R} \vee \mathrm{L}$, where $\mathrm{R}$ and $\mathrm{L}$ are the pseudovarieties of semigroups of all $\mathcal{R}$-trivial semigroups and of all $\mathcal{L}$-trivial semigroups, respectively, is not finitely based. Another contribution to the resolution of Pin's problem was given by the author [17] who showed that $\mathrm{O}$ contains all semidirect products of a chain (considered as a semilattice) by a semigroup of injective order-preserving partial transformations on a finite chain. Nevertheless, Pin's question is still unanswered.

The pseudovariety OP generated by all semigroups of orientation-preserving full transformations on a finite chain was studied by Catarino and Higgins in [6]. They showed that $\mathrm{OP}$ is self-dual and contains the join of $\mathrm{O}$ with the pseudovariety of all finite commutative monoids. Moreover, Catarino and Higgins also proved in [6] that the interval between these two pseudovarieties contains a chain of pseudovarieties isomorphic to the chain of real numbers. A division theorem for OP was presented by the author in [16]. He proved that the pseudovariety POPI generated by all semigroups of injective orientation-preserving partial transformations on a finite chain is a (proper) subpseudovariety of OP.

Semigroups of order-preserving transformations have long been considered in the literature. In 1962, Aǐzenštat [2] and Popova [26] exhibited presentations for $\mathcal{O}_{n}$, the monoid of all order-preserving full transformations on a chain with $n$ elements, and for $\mathcal{P} \mathcal{O}_{n}$, the monoid of all order-preserving partial transformations on a chain with $n$ elements. Some years later, in 1971, Howie [24] studied some combinatorial and algebraic properties of $\mathcal{O}_{n}$ and, in 1992, Gomes and Howie [22] revisited the monoids $\mathcal{O}_{n}$ and $\mathcal{P} \mathcal{O}_{n}$. More recently, the injective counterpart of $\mathcal{O}_{n}$, i.e. the monoid $\mathcal{P O} \mathcal{I}_{n}$ of all injective members of $\mathcal{P} \mathcal{O}_{n}$, has been object of study by the author in several papers $[11,12,14,15,17]$ and also by Cowan and Reilly [8].

On the other hand, the notion of an orientation-preserving transformation was introduced by McAlister in [25] and, independently, by Catarino and Higgins in [5]. The monoid $\mathcal{O P}_{n}$, of all orientation-preserving full transformations on a chain with $n$ elements, was also considered by Catarino in [4] and by Arthur and Ruškuc in [3]. The injective counterpart of $\mathcal{O P}_{n}$, i.e. the monoid $\mathcal{P O P} \mathcal{I}_{n}$ of all injective orientation-preserving partial transformations on a chain with $n$ elements, was studied by the first author in $[13,16]$.

Recently, the author together with Gomes and Jesus [18] exhibited presentations for the monoids $\mathcal{P O D \mathcal { I } _ { n }}$ of all injective order-preserving or order-reversing partial transformations on a chain with $n$ elements, and for the monoid $\mathcal{P O} \mathcal{R} \mathcal{I}_{n}$ of all injective orientationpreserving or orientation-reversing partial transformations on a chain with $n$ elements. The same authors in [19] also gave presentations for the monoid $\mathcal{O D}_{n}$ of all order-preserving or order-reversing full transformations on a chain with $n$ element; for the monoid $\mathcal{P} \mathcal{O D}_{n}$ of all order-preserving or order-reversing partial transformations on a chain with $n$ elements; for the monoid $\mathcal{P O} \mathcal{P}_{n}$ of all orientation-preserving partial transformations on a chain with $n$ elements; and for the monoid $\mathcal{P O} \mathcal{R}_{n}$ of all orientation-preserving or orientation-reversing partial transformations on a chain with $n$ elements. The lattice of the congruences of some 
of these monoids were studied in $[20,21]$ by the same authors.

Together with Delgado the author $[9,10]$ have computed the abelian kernels of the monoids $\mathcal{P O} \mathcal{I}_{n}, \mathcal{P O P} \mathcal{I}_{n}, \mathcal{P O D} \mathcal{I}_{n}$ and $\mathcal{P O R} \mathcal{I}_{n}$. More recently, the same authors together with Cordeiro determined all relative abelian kernels of these four monoids [7].

Next, we will introduce or precise some definitions.

Denote by $\mathcal{P} \mathcal{T}_{n}\left[\mathcal{T}_{n}\right]$ the monoid of all partial [full] transformations of a set with $n$ elements, say $X_{n}=\{1,2, \ldots, n\}$, and by $\mathcal{I}_{n}$ the symmetric inverse monoid, i.e. the submonoid of $\mathcal{P} \mathcal{T}_{n}$ of all injective (partial) transformations of $X_{n}$.

From now on, we consider $X_{n}$ as a chain with the usual order: $X_{n}=\{1<2<\cdots<n\}$.

We say that a transformation $s$ in $\mathcal{P} \mathcal{T}_{n}$ is order-preserving [order-reversing] if, for all $x, y \in \operatorname{Dom}(s), x \leq y$ implies $x s \leq y s[x s \geq y s]$. Clearly, the product of two orderpreserving transformations or of two order-reversing transformations is order-preserving and the product of an order-preserving transformation by an order-reversing transformation is order-reversing.

Denote by $\mathcal{O}_{n}\left[\mathcal{P O} \mathcal{I}_{n}\right]$ the submonoid of $\mathcal{I}_{n}\left[\mathcal{I}_{n}\right]$ whose elements are order-preserving and by $\mathcal{O D}_{n}\left[\mathcal{P O D} \mathcal{I}_{n}\right]$ the submonoid of $\mathcal{T}_{n}\left[\mathcal{I}_{n}\right]$ whose elements are either order-preserving or order-reversing.

Next, let $a=\left(a_{1}, a_{2}, \ldots, a_{t}\right)$ be a sequence of $t(t \geq 0)$ elements from the chain $X_{n}$. We say that $a$ is cyclic [anti-cyclic] if there exists no more than one index $i \in$ $\{1, \ldots, t\}$ such that $a_{i}>a_{i+1}\left[a_{i}<a_{i+1}\right]$, where $a_{t+1}$ denotes $a_{1}$. Let $s \in \mathcal{P} \mathcal{T}_{n}$ and suppose that $\operatorname{Dom}(s)=\left\{a_{1}, \ldots, a_{t}\right\}$, with $t \geq 0$ and $a_{1}<\cdots<a_{t}$. We say that $s$ is an orientation-preserving [orientation-reversing] transformation if the sequence of its images $\left(a_{1} s, \ldots, a_{t} s\right)$ is cyclic [anti-cyclic]. It is also clear that the product of two orientationpreserving or of two orientation-reversing transformations is orientation-preserving and the product of an orientation-preserving transformation by an orientation-reversing transformation is orientation-reversing.

Denote by $\mathcal{O P} \mathcal{P}_{n}\left[\mathcal{P O P} \mathcal{I}_{n}\right]$ the submonoid of $\mathcal{I}_{n}\left[\mathcal{I}_{n}\right]$ whose elements are orientationpreserving and by $\mathcal{O} \mathcal{R}_{n}\left[\mathcal{P O} \mathcal{R} \mathcal{I}_{n}\right]$ the submonoid of $\mathcal{T}_{n}\left[\mathcal{I}_{n}\right]$ whose elements are either orientation-preserving or orientation-reversing.

The following diagram, with respect to the inclusion relation, clarifies the relationship between these various monoids:

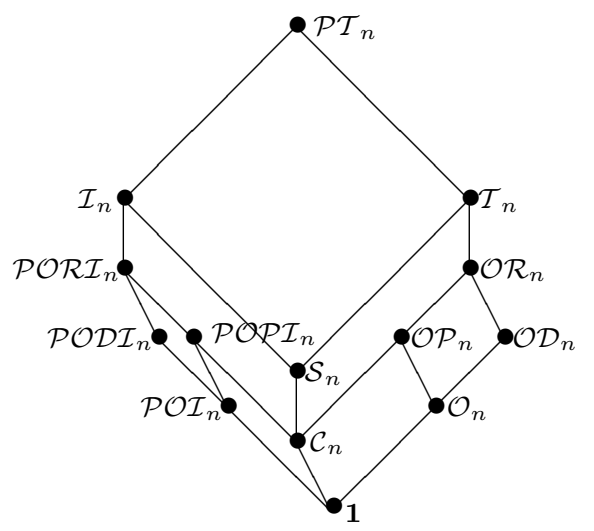

( 1 denotes the trivial monoid, $\mathcal{C}_{n}$ the cyclic group of order $n$ and $\mathcal{S}_{n}$ the symmetric group on $\left.X_{n}\right)$. 
Recall that a pseudovariety of semigroups is a class of finite semigroups closed under formation of finite direct products, subsemigroups and homomorphic images.

Let $S$ and $T$ be two semigroups. A division of semigroups $\tau: S \longrightarrow T$ is a relation from $S$ into $T$ (i.e. a function from $S$ into the power set of $T$ ) such that:

- For all $s \in S,(s) \tau \neq \emptyset$, i.e. $s$ is totally defined;

- For all $s_{1}, s_{2} \in S,\left(s_{1}\right) \tau\left(s_{2}\right) \tau \subseteq\left(s_{1} s_{2}\right) \tau$, i.e. $s$ is a relation of semigroups; and

- For all $s_{1}, s_{2} \in S,\left(s_{1}\right) \tau \cap\left(s_{2}\right) \tau \neq \emptyset \Longrightarrow s_{1}=s_{2}$, i.e. $s$ is injective.

We say that $S$ divides $T$ if there exists a division of semigroups $\tau: S \longrightarrow T$.

Notice that, given a family $\mathcal{X}$ of finite semigroups, it is easy to show that the pseudovariety of semigroups generated by $\mathcal{X}$ is the class of all semigroups that divide a finite direct product of members of $\mathcal{X}$.

With the above notation, we have that:

- $\mathrm{O}$ is the pseudovariety of semigroups generated by $\left\{\mathcal{O}_{n} \mid n \in \mathbb{N}\right\}$;

- POI is the pseudovariety of semigroups generated by $\left\{\mathcal{P O} \mathcal{I}_{n} \mid n \in \mathbb{N}\right\}$;

- OP is the pseudovariety of semigroups generated by $\left\{\mathcal{O} \mathcal{P}_{n} \mid n \in \mathbb{N}\right\}$; and

- POPI is the pseudovariety of semigroups generated by $\left\{\mathcal{P O P} \mathcal{I}_{n} \mid n \in \mathbb{N}\right\}$.

Next, also define:

- OD as the pseudovariety of semigroups generated by $\left\{\mathcal{O D}_{n} \mid n \in \mathbb{N}\right\}$;

- PODI as the pseudovariety of semigroups generated by $\left\{\mathcal{P O D} \mathcal{I}_{n} \mid n \in \mathbb{N}\right\}$;

- OR as the pseudovariety of semigroups generated by $\left\{\mathcal{O} \mathcal{R}_{n} \mid n \in \mathbb{N}\right\}$; and

- PORI as the pseudovariety of semigroups generated by $\left\{\mathcal{P O} \mathcal{R} \mathcal{I}_{n} \mid n \in \mathbb{N}\right\}$.

Now, we can state the main results of this paper, which are the analogues of the result presented by the author in [11] (and in [16]).

Theorem 1 Every semigroup of injective order-preserving or order-reversing partial transformations on a finite chain belongs to OD.

Theorem 2 Every semigroup of injective orientation-preserving or orientation-reversing partial transformations on a finite chain belongs to OR. 


\section{The proofs}

Let $X$ be a finite set and let $Y$ be a subset of $X$. Denote by $\mathcal{P} \mathcal{T}(X)[\mathcal{P} \mathcal{T}(Y)]$ the monoid of all partial transformations of $X[Y]$. Let $S$ be a subsemigroup of $\mathcal{P} \mathcal{T}(Y)$ and let $T$ be a subsemigroup of $\mathcal{P} \mathcal{T}(X)$. Define a relation $\tau: S \longrightarrow T$ by

$$
(s) \tau=\left\{t \in T \mid Y t^{-1} \subseteq Y \text { and }\left.t\right|_{Y t^{-1}}=s\right\},
$$

for all $s \in S$. Notice that, $Y t^{-1}$ denotes the set $\{x \in \operatorname{Dom}(t) \mid(x) t \in Y\}$ and $\left.t\right|_{Y t^{-1}}$ the restriction of the map $t$ to the set $Y t^{-1}$. Hence, we have:

Proposition 2.1 With the foregoing, $\tau: S \longrightarrow T$ is an injective relation of semigroups. Moreover, if $\tau$ is completely defined then $S$ divides $T$.

Proof. First, notice that $\tau$ is clearly an injective relation. Indeed, given $s_{1}, s_{2} \in S$ such that $\left(s_{1}\right) \tau \cap\left(s_{2}\right) \tau \neq \emptyset$, we can take $t \in\left(s_{1}\right) \tau \cap\left(s_{2}\right) \tau$ and so, in particular, we have $s_{1}=\left.t\right|_{Y t^{-1}}=s_{2}$.

Now, let $s_{1}, s_{2} \in S$. We will prove that $\left(s_{1}\right) \tau\left(s_{2}\right) \tau \subseteq\left(s_{1} s_{2}\right) \tau$. If $\left(s_{1}\right) \tau=\emptyset$ or $\left(s_{2}\right) \tau=\emptyset$ then this inclusion is obvious. Thus, we can suppose that $\left(s_{1}\right) \tau \neq \emptyset$ and $\left(s_{2}\right) \tau \neq \emptyset$.

Let $t_{1} \in\left(s_{1}\right) \tau$ and $t_{2} \in\left(s_{2}\right) \tau$. Then $Y t_{1}^{-1} \subseteq Y, Y t_{2}^{-1} \subseteq Y,\left.t_{1}\right|_{Y t_{1}^{-1}}=s_{1}$ and $\left.t_{2}\right|_{Y t_{2}^{-1}}=s_{2}$. In order to prove that $t_{1} t_{2} \in\left(s_{1} s_{2}\right) \tau$, we must show that $Y\left(t_{1} t_{2}\right)^{-1} \subseteq Y$ and $\left.\left(t_{1} t_{2}\right)\right|_{Y\left(t_{1} t_{2}\right)^{-1}}=$ $s_{1} s_{2}$.

Regarding the first condition, let $x \in Y\left(t_{1} t_{2}\right)^{-1}$. Then $(x)\left(t_{1} t_{2}\right) \in Y$, i.e. $\left((x) t_{1}\right) t_{2} \in Y$, whence $(x) t_{1} \in Y t_{2}^{-1} \subseteq Y$, i.e. $(x) t_{1} \in Y$. It follows that $x \in Y t_{1}^{-1} \subseteq Y$ and so $x \in Y$. Hence, $Y\left(t_{1} t_{2}\right)^{-1} \subseteq Y$.

Next, we want to show that $\left.\left(t_{1} t_{2}\right)\right|_{Y\left(t_{1} t_{2}\right)^{-1}}=s_{1} s_{2}$. We begin by proving that $\left.\left(t_{1} t_{2}\right)\right|_{Y\left(t_{1} t_{2}\right)^{-1}}$ and $s_{1} s_{2}$ have the same domain. Let $x \in \operatorname{Dom}\left(s_{1} s_{2}\right)$. Then $x \in \operatorname{Dom}\left(s_{1}\right)=\operatorname{Dom}\left(t_{1}\right) \cap$ $Y t_{1}^{-1}$ and $(x) s_{1} \in \operatorname{Dom}\left(s_{2}\right)=\operatorname{Dom}\left(t_{2}\right) \cap Y t_{2}^{-1}$. Thus $(x) t_{1}=(x) s_{1}$, whence $(x) t_{1} \in$ $\operatorname{Dom}\left(t_{2}\right) \cap Y t_{2}^{-1}$ and so $\left((x) t_{1}\right) t_{2} \in Y$, i.e. $x \in Y\left(t_{1} t_{2}\right)^{-1}$. Hence $x \in \operatorname{Dom}\left(t_{1} t_{2}\right) \cap$ $Y\left(t_{1} t_{2}\right)^{-1}=\operatorname{Dom}\left(\left.\left(t_{1} t_{2}\right)\right|_{Y\left(t_{1} t_{2}\right)^{-1}}\right)$ and so $\operatorname{Dom}\left(s_{1} s_{2}\right) \subseteq \operatorname{Dom}\left(\left.\left(t_{1} t_{2}\right)\right|_{Y\left(t_{1} t_{2}\right)^{-1}}\right)$. Conversely,

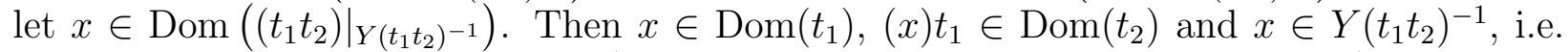
$(x)\left(t_{1} t_{2}\right) \in Y$. Hence $(x) t_{1} \in Y t_{2}^{-1} \subseteq Y$ and so $(x) t_{1} \in Y$, whence $x \in Y t_{1}^{-1}$. Then, we have $x \in \operatorname{Dom}\left(t_{1}\right) \cap Y t_{1}^{-1}=\operatorname{Dom}\left(s_{1}\right)$, from which it follows that $(x) t_{1}=(x) s_{1}$, and so $(x) s_{1} \in \operatorname{Dom}\left(t_{2}\right) \cap Y t_{2}^{-1}=\operatorname{Dom}\left(s_{2}\right)$. Thus $x \in \operatorname{Dom}\left(s_{1} s_{2}\right)$ and so $\operatorname{Dom}\left(\left.\left(t_{1} t_{2}\right)\right|_{Y\left(t_{1} t_{2}\right)^{-1}}\right) \subseteq$ $\operatorname{Dom}\left(s_{1} s_{2}\right)$. Hence $\operatorname{Dom}\left(s_{1} s_{2}\right)=\operatorname{Dom}\left(\left.\left(t_{1} t_{2}\right)\right|_{Y\left(t_{1} t_{2}\right)^{-1}}\right)$. Finally, if $x \in \operatorname{Dom}\left(s_{1} s_{2}\right)$ then $x \in \operatorname{Dom}\left(s_{1}\right)$ and $(x) s_{1} \in \operatorname{Dom}\left(s_{2}\right)$, whence $x \in \operatorname{Dom}\left(t_{1}\right)$ and $(x) t_{1}=(x) s_{1} \in \operatorname{Dom}\left(t_{2}\right)$ and so $(x)\left(t_{1} t_{2}\right)=\left((x) t_{1}\right) t_{2}=\left((x) t_{1}\right) s_{2}=\left((x) s_{1}\right) s_{2}=(x)\left(s_{1} s_{2}\right)$. Therefore $\left.\left(t_{1} t_{2}\right)\right|_{Y\left(t_{1} t_{2}\right)^{-1}}=s_{1} s_{2}$, as required.

\section{The proof of Theorem 1}

Let us consider the chain $X=\{\overline{0}<1<\overline{1}<2<\overline{2}<\cdots<n<\bar{n}\}$ (with $2 n+1$ elements) and its subchain $Y=\{1<2<\cdots<n\}$. Consider the semigroups $\mathcal{O D}_{2 n+1}$ and $\mathcal{P O D D} \mathcal{I}_{n}$ built over $X$ and $Y$, respectively, and the relation $\tau: \mathcal{P O D} \mathcal{I}_{n} \longrightarrow \mathcal{O D}_{2 n+1}$ defined by

$$
(s) \tau=\left\{t \in \mathcal{O D}_{2 n+1} \mid Y t^{-1} \subseteq Y \text { and }\left.t\right|_{Y t^{-1}}=s\right\},
$$


for all $s \in \mathcal{P} \mathcal{O D} \mathcal{I}_{n}$. We claim that $\tau$ is completely defined.

Indeed, for an element $s \in \mathcal{P O D} \mathcal{I}_{n}$ such that $\operatorname{Dom}(s)=\left\{i_{1}<i_{2}<\cdots<i_{k}\right\}(1 \leq k \leq n)$, define $\bar{s} \in \mathcal{O D}_{2 n+1}$ by

$$
(x) \bar{s}= \begin{cases}\overline{0} & \text { if } \overline{0} \leq x<i_{1} \\ \overline{\left(i_{p}\right) s} & \text { if } x=i_{p}, \text { for some } 1 \leq p \leq k \\ \overline{\left(i_{p}\right) s} & \text { if } i_{p}<x<i_{p+1}, \text { for some } 1 \leq p \leq k-1 \\ \bar{n} & \text { if } i_{k}<x \leq \bar{n}\end{cases}
$$

if $s$ is order-preserving, and by

$$
(x) \bar{s}= \begin{cases}\bar{n} & \text { if } \overline{0} \leq x<i_{1} \\ \frac{\left(i_{p}\right) s}{\left(i_{p+1}\right) s} & \text { if } x=i_{p}, \text { for some } 1 \leq p \leq k \\ \overline{0} & \text { if } i_{p}<x<i_{p+1}, \text { for some } 1 \leq p \leq k-1 \\ \text { if } i_{k}<x \leq \bar{n}\end{cases}
$$

if $s$ is order-reversing and $k \geq 2$. If $s \in \mathcal{P O D} \mathcal{I}_{n}$ is the empty transformation, then define $\bar{s}$ as the constant transformation of $\mathcal{O D}_{2 n+1}$ with image $\{\overline{0}\}$.

Examples 2.2 Let $n=7$. Then:

- If $s=\left(\begin{array}{ccc}2 & 4 & 5 \\ 1 & 2 & 7\end{array}\right)$ then $\bar{s}=\left(\begin{array}{ccccccccccccccc}\overline{0} & 1 & \overline{1} & \mathbf{2} & \overline{2} & 3 & \overline{3} & \mathbf{4} & \overline{4} & \mathbf{5} & \overline{5} & 6 & \overline{6} & 7 & \overline{7} \\ \overline{0} & \overline{0} & \overline{0} & \mathbf{1} & \overline{1} & \overline{1} & \overline{1} & \mathbf{2} & \overline{2} & \mathbf{7} & \overline{7} & \overline{7} & \overline{7} & \overline{7} & \overline{7}\end{array}\right)$;

- If $s=\left(\begin{array}{lll}2 & 4 & 5 \\ 7 & 2 & 1\end{array}\right)$ then $\bar{s}=\left(\begin{array}{lllllllllllllll}\overline{0} & 1 & \overline{1} & \mathbf{2} & \overline{2} & 3 & \overline{3} & \mathbf{4} & \overline{4} & \mathbf{5} & \overline{5} & 6 & \overline{6} & 7 & \overline{7} \\ \overline{7} & \overline{7} & \overline{7} & \mathbf{7} & \overline{2} & \overline{2} & \overline{2} & \mathbf{2} & \overline{1} & \mathbf{1} & \overline{0} & \overline{0} & \overline{0} & \overline{0} & \overline{0}\end{array}\right)$.

It is clear that $\bar{s} \in(s) \tau$, for all $s \in \mathcal{P} \mathcal{O D} \mathcal{I}_{n}$. Thus, by Proposition 2.1, we have:

Theorem 2.3 The semigroup $\mathcal{P O D} \mathcal{I}_{n}$ divides $\mathcal{O D}_{2 n+1}$.

Now, as a corollary, we obtain (the following reformulation of) Theorem 1:

\section{Corollary 2.4 PODI $\subset$ OD.}

Notice that, since PODI is generated by inverse semigroups, all elements of PODI have commuting idempotents. On the other hand, it is clear that, for instance, $\mathcal{O D}_{2}$ has noncommuting idempotents. Therefore, the inclusion PODI $\subset$ OD is strict.

We remark that, as $\bar{s}$ is order-preserving when $s$ is order-preserving, by simply adapting the definition of $\tau$ to order-preserving transformations only, we recover the result presented by the author in [11]: POI $\subset \mathrm{O}$. 


\section{The proof of Theorem 2}

Now, we consider the chain $X=\{1<\overline{1}<2<\overline{2}<\cdots<n<\bar{n}\}$ (with $2 n$ elements) and its subchain $Y=\{1<2<\cdots<n\}$. Also, we consider the semigroups $\mathcal{O R}_{2 n}$ and $\mathcal{P} \mathcal{O} \mathcal{R} \mathcal{I}_{n}$ built over $X$ and $Y$, respectively, and the relation $\tau: \mathcal{P O} \mathcal{R} \mathcal{I}_{n} \longrightarrow \mathcal{O R}_{2 n}$ defined by

$$
(s) \tau=\left\{t \in \mathcal{O R}_{2 n} \mid Y t^{-1} \subseteq Y \text { and }\left.t\right|_{Y t^{-1}}=s\right\},
$$

for all $s \in \mathcal{P} \mathcal{O R} \mathcal{I}_{n}$. Again, we will prove that $\tau$ is completely defined.

Let $s \in \mathcal{P O D} \mathcal{I}_{n}$ be such that $\operatorname{Dom}(s)=\left\{i_{1}<i_{2}<\cdots<i_{k}\right\}$, with $1 \leq k \leq n$. If $s$ is orientation-preserving, we define $\bar{s} \in \mathcal{O R}_{2 n}$ by

$$
(x) \bar{s}= \begin{cases}\frac{\left(i_{p}\right) s}{\left(i_{p}\right) s} & \text { if } x=i_{p}, \text { for some } 1 \leq p \leq k \\ \frac{\left.i_{k}\right) s}{\left(i_{k}\right.} & \text { if } \overline{1} \leq x<i_{p+1}, \text { for some } 1 \leq p \leq k-1 \\ i_{k}<x \leq \bar{n}\end{cases}
$$

If $s$ is orientation-reversing and $k \geq 3$, we define $\bar{s} \in \mathcal{O} \mathcal{R}_{2 n}$ by

$$
(x) \bar{s}= \begin{cases}\frac{\left(i_{p}\right) s}{\left(i_{p+1}\right) s} & \text { if } x=i_{p}, \text { for some } 1 \leq p \leq k \\ \frac{\text { if } i_{p}<x<i_{p+1}, \text { for some } 1 \leq p \leq k-1}{\left(i_{1}\right) s} & \text { if } \overline{1} \leq x<i_{1} \text { or } i_{k}<x \leq \bar{n}\end{cases}
$$

Finally, if $s \in \mathcal{P O} \mathcal{R} \mathcal{I}_{n}$ is the empty transformation, then define $\bar{s}$ as the constant transformation of $\mathcal{O R}_{2 n}$ with image $\{\overline{1}\}$.

Examples 2.5 Let $n=7$. Then:

- If $s=\left(\begin{array}{lll}2 & 4 & 5 \\ 1 & 2 & 7\end{array}\right)$ then $\bar{s}=\left(\begin{array}{cccccccccccccc}1 & \overline{1} & \mathbf{2} & \overline{2} & 3 & \overline{3} & \mathbf{4} & \overline{4} & \mathbf{5} & \overline{5} & 6 & \overline{6} & 7 & \overline{7} \\ \overline{7} & \overline{7} & \mathbf{1} & \overline{1} & \overline{1} & \overline{1} & \mathbf{2} & \overline{2} & \mathbf{7} & \overline{7} & \overline{7} & \overline{7} & \overline{7} & \overline{7}\end{array}\right)$;

- If $s=\left(\begin{array}{lll}2 & 4 & 5 \\ 7 & 2 & 1\end{array}\right)$ then $\bar{s}=\left(\begin{array}{llllllllllllll}1 & \overline{1} & \mathbf{2} & \overline{2} & 3 & \overline{3} & \mathbf{4} & \overline{4} & \mathbf{5} & \overline{5} & 6 & \overline{6} & 7 & \overline{7} \\ \overline{7} & \overline{7} & \mathbf{7} & \overline{2} & \overline{2} & \overline{2} & \mathbf{2} & \overline{1} & \mathbf{1} & \overline{7} & \overline{7} & \overline{7} & \overline{7} & \overline{7}\end{array}\right)$;

- If $s=\left(\begin{array}{lll}2 & 4 & 5 \\ 7 & 1 & 2\end{array}\right)$ then $\bar{s}=\left(\begin{array}{llllllllllllll}1 & \overline{1} & \mathbf{2} & \overline{2} & 3 & \overline{3} & \mathbf{4} & \overline{4} & \mathbf{5} & \overline{5} & 6 & \overline{6} & 7 & \overline{7} \\ \overline{2} & \overline{2} & \mathbf{7} & \overline{7} & \overline{7} & \overline{7} & \mathbf{1} & \overline{1} & \mathbf{2} & \overline{2} & \overline{2} & \overline{2} & \overline{2} & \overline{2}\end{array}\right)$;

- If $s=\left(\begin{array}{lll}2 & 4 & 5 \\ 2 & 1 & 7\end{array}\right)$ then $\bar{s}=\left(\begin{array}{cccccccccccccc}1 & \overline{1} & \mathbf{2} & \overline{2} & 3 & \overline{3} & \mathbf{4} & \overline{4} & \mathbf{5} & \overline{5} & 6 & \overline{6} & 7 & \overline{7} \\ \overline{2} & \overline{2} & \mathbf{2} & \overline{1} & \overline{1} & \overline{1} & \mathbf{1} & \overline{7} & \mathbf{7} & \overline{2} & \overline{2} & \overline{2} & \overline{2} & \overline{2}\end{array}\right)$;

- If $s=\left(\begin{array}{lll}1 & 4 & 5 \\ 2 & 6 & 1\end{array}\right)$ then $\bar{s}=\left(\begin{array}{llllllllllllll}\mathbf{1} & \overline{1} & 2 & \overline{2} & 3 & \overline{3} & \mathbf{4} & \overline{4} & \mathbf{5} & \overline{5} & 6 & \overline{6} & 7 & \overline{7} \\ \mathbf{2} & \overline{2} & \overline{2} & \overline{2} & \overline{2} & \overline{2} & \mathbf{6} & \overline{6} & \mathbf{1} & \overline{1} & \overline{1} & \overline{1} & \overline{1} & \overline{1}\end{array}\right)$;

- If $s=\left(\begin{array}{lll}1 & 4 & 5 \\ 1 & 6 & 3\end{array}\right)$ then $\bar{s}=\left(\begin{array}{llllllllllllll}\mathbf{1} & \overline{1} & 2 & \overline{2} & 3 & \overline{3} & \mathbf{4} & \overline{4} & \mathbf{5} & \overline{5} & 6 & \overline{6} & 7 & \overline{7} \\ \mathbf{1} & \overline{6} & \overline{6} & \overline{6} & \overline{6} & \overline{6} & \mathbf{6} & \overline{3} & \mathbf{3} & \overline{1} & \overline{1} & \overline{1} & \overline{1} & \overline{1}\end{array}\right)$.

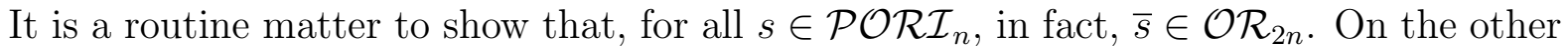
hand, clearly $\bar{s} \in(s) \tau$, for all $s \in \mathcal{P} \mathcal{O} \mathcal{R} \mathcal{I}_{n}$. Now, applying Proposition 2.1, we also have:

Theorem 2.6 The semigroup $\mathcal{P O R} \mathcal{I}_{n}$ divides $\mathcal{O R}_{2 n}$. 
Consequently, we deduce Theorem 2 (reformulated as):

Corollary 2.7 PORI $\subset$ OR.

Notice that, likewise for the order case, the inclusion PORI $\subset \mathrm{OR}$ is also strict.

Observe also that, as $\bar{s}$ is orientation-preserving when $s$ is orientation-preserving, again by simply adapting the definition of $\tau$ to just orientation-preserving transformations, we obtain that POPI $\subset$ OP [16].

\section{References}

[1] Almeida, J., and M.V. Volkov, The gap between partial and full, Int. J. Algebra Comput. Vol. 8, No. 3 (1998), 399-430.

[2] A.Ya. Aǔzenštat, The defining relations of the endomorphism semigroup of a finite linearly ordered set, Sibirsk. Mat. 3 (1962), 161-169 (Russian).

[3] R.E. Arthur and N. Ruškuc, Presentations for two extensions of the monoid of orderpreserving mappings on a finite chain, Southeast Asian Bull. Math. 24 (2000), 1-7.

[4] P.M. Catarino, Monoids of orientation-preserving transformations of a finite chain and their presentations, Semigroups and Applications, eds. J.M. Howie and N. Ruškuc, World Scientific, (1998), 39-46.

[5] P.M. Catarino and P.M. Higgins, The monoid of orientation-preserving mappings on a chain, Semigroup Forum 58 (1999), 190-206.

[6] P.M. Catarino and P.M. Higgins, The pseudovariety generated by all semigroups of orientation-preserving transformations on a finite cycle, Internat. J. Algebra Comput.12 (2002), no. 3, 387-405.

[7] E. Cordeiro, M. Delgado and V.H. Fernandes, Relative abelian kernels of some classes of transformation monoids, Submitted.

[8] D.F. Cowan and N.R. Reilly, Partial cross-sections of symmetric inverse semigroups, Int. J. Algebra Comput. 5 (1995) 259-287.

[9] M. Delgado and V.H. Fernandes, Abelian kernels of some monoids of injective partial transformations and an application, Semigroup Forum 61 (2000) 435-452.

[10] M. Delgado and V.H. Fernandes, Abelian kernels of monoids of order-preserving maps and of some of its extensions. Semigroup Forum 68 (2004), 335-356

[11] V.H. Fernandes, Semigroups of order-preserving mappings on a finite chain: a new class of divisors, Semigroup Forum 54 (1997), 230-236.

[12] V.H. Fernandes, Normally ordered inverse semigroups, Semigroup Forum 58 (1998) 418-433. 
[13] V.H. Fernandes, The monoid of all injective orientation preserving partial transformations on a finite chain, Comm. Algebra 28 (2000), 3401-3426.

[14] V.H. Fernandes, The monoid of all injective order preserving partial transformations on a finite chain, Semigroup Forum 62 (2001), 178-204.

[15] V.H. Fernandes, Presentations for some monoids of partial transformations on a finite chain: a survey, Semigroups, Algorithms, Automata and Languages, eds. Gracinda M. S. Gomes \& Jean-Éric Pin \& Pedro V. Silva, World Scientific, (2002), 363-378.

[16] V.H. Fernandes, A division theorem for the pseudovariety generated by semigroups of orientation preserving transformations on a finite chain, Comm. Algebra 29 (2001) 451-456.

[17] V.H. Fernandes, Semigroups of order-preserving mappings on a finite chain: another class of divisors, Izvestiya VUZ. Matematika 3 (478) (2002) 51-59 (Russian).

[18] V.H. Fernandes, G.M.S. Gomes and M.M. Jesus, Presentations for some monoids of injective partial transformations on a finite chain, Southeast Asian Bull. Math. 28 (2004), 903-918.

[19] V.H. Fernandes, G.M.S. Gomes and M.M. Jesus, Presentations for some monoids of partial transformations on a finite chain, Comm. Algebra 33 (2005), 587-604.

[20] V.H. Fernandes, G.M.S. Gomes and M.M. Jesus, Congruences on monoids of orderpreserving or order-reversing transformations on a finite chain, Glasgow Mathematical Journal 47 (2005), 413-424.

[21] V.H. Fernandes, G.M.S. Gomes and M.M. Jesus, Congruences on monoids of transformations preserving the orientation on a finite chain, Submitted.

[22] G.M.S. Gomes and J.M. Howie, On the ranks of certain semigroups of order-preserving transformations, Semigroup Forum 45 (1992), 272-282.

[23] P.M. Higgins, Divisors of semigroups of order-preserving mappings on a finite chain, Int. J. Algebra Comput. 5 (1995), 725-742.

[24] J.M. Howie, Product of idempotents in certain semigroups of transformations, Proc. Edinburgh Math. Soc. 17 (1971) 223-236.

[25] D. McAlister, Semigroups generated by a group and an idempotent, Comm. Algebra 26 (1998), 515-547.

[26] L.M. Popova, The defining relations of the semigroup of partial endomorphisms of a finite linearly ordered set, Leningradskij gosudarstvennyj pedagogicheskij institut imeni A. I. Gerzena, Uchenye Zapiski 238 (1962) 78-88 (Russian).

[27] V.B. Repnitskiul and M.V. Volkov, The finite basis problem for the pseudovariety O, Proc. R. Soc. Edinb., Sect. A, Math. 128, No.3 (1998), 661-669. 
[28] A. Vernitskii and M.V. Volkov, A proof and generalisation of Higgins' division theorem for semigroups of order-preserving mappings, Izv.vuzov. Matematika, (1995), No.1, 3844.

Author's second address:

Centro de Álgebra da Universidade de Lisboa

Av. Prof. Gama Pinto, 2

1649-003 Lisboa

Portugal 